\title{
Perioperative blood transfusion is associated with poorer survival in patients with gastric cancer
}

\author{
Xiaohua Tu ${ }^{\wedge}$, Yan Zang ${ }^{\wedge}$, Haihui Gu, Huijun Guo, Xin Ye, Zhanshan Cha ${ }^{*}$, Baohua Qian \\ Department of Transfusion, Changhai Hospital, Shanghai 200433 China
}

\begin{abstract}
The putative prognostic significance of perioperative blood transfusions on gastric cancers is controversial and the published results are contradictory. The aim of this study was to evaluate the prognostic influence of transfusion on Chinese gastric cancer surgery. Six hundreds and seventy-six patients who underwent curative gastrectomy for gastric cancer from 2000 to 2004 were retrospectively reviewed. Uni- and multivariate analyses of the incidence and amount of transfusion, and a comparison of the clinicopathological features were performed. Subgroup analyses of prognosis according to stage, tumor size, and pretreatment anemia were carried out. Blood transfusion was significantly associated with older age ( $>60$ year), larger tumor $(>6 \mathrm{~cm})$, upper and middle location, surgical margin status, and pretreatment anemia. In addition, tumors in the transfused group were more advanced in depth of invasion, nodal stage, and TNM stage. No significant relationship was found between the amount of transfused blood and prognosis. Subgroup analyses of prognosis according to stage showed significant differences in stages II and III , between the transfused and nontransfused groups. Significant difference between the transfused and nontransfused groups could be observed in two subgroups of tumor size. Patients with or without anemia in the nontransfused group both had a longer survival time than those in the transfused group. On multivariate analysis, transfusion was shown to be an independent risk factor for poor prognosis. This study suggests that perioperative blood transfusion is associated with a significantly worse prognosis following gastric cancer surgery. The parameters such as advanced stage, tumor size, and anemia do not affect its prognostic value.
\end{abstract}

Keywords: gastric cancer, perioperative blood transfusion, survival analysis

\section{INTRODUCTION}

The relationship between blood transfusion and immunological changes or immune modulation has been debated for over thirty years ${ }^{[1,2]}$. The results, however, have been contradictory since blood transfusion is beneficial to some patients, but harmful to

\footnotetext{
*Correspondence to: Zhanshan Cha, Department of Transfusion, Changhai Hospital, Shanghai 200433, China. Tel: 86-21-31162122, Fax: 86-21-81873299, E-mail: chazhanshan@163.com; Baohua Qian, Department of Transfusion, Changhai Hospital, Shanghai 200433, China. Tel: 86-21-31162121, Fax: 86-21-81873299, E-mail: qianbh1963@163.com.

${ }^{\Delta}$ These authors contributed equally to the work.
}

others. Previous experimental and clinical studies showed that perioperative blood transfusions could induce immunosuppression, which has a direct adverse effect on cancer recurrence and results in a poor prognosis for patients with malignant tumors, including hepatocellular carcinoma ${ }^{[3]}$, breast cancer ${ }^{[4]}$, head and neck cancer ${ }^{[5]}$, lung cancer ${ }^{[6]}$, osteosarcoma ${ }^{[7]}$, esophageal cancer ${ }^{[8]}$, and prostate cancer ${ }^{[9]}$. In gastric cancer, however, the putative prognostic significance of perioperative blood transfusions is controversial and the published results are contradictory. According to published reports, perioperative blood transfusion can be regarded as an independent prognostic factor for patients with gastric carcinoma ${ }^{[10-15]}$, whereas other studies do not reveal the prognostic value of periop- 
erative blood transfusions in gastric carcinoma ${ }^{[16-18]}$. The latter believed that cases needing transfusion were more advanced or operatively difficult, and thus they didn't regard transfusion as an independent predictor.

In this study, we performed a retrospective study to investigate whether perioperative blood transfusion significantly affects survival in patients undergoing gastric resection for gastric cancer. Our results found a significant relationship between advanced stage tumor and perioperative blood transfusion. Further analysis suggested that blood transfusion itself seemed to be an unfavorable prognostic factor for gastric cancer patients, especially for those in stage II and III .

\section{MATERIALS AND METHODS}

\section{Study population}

Six hundreds and seventy-six gastric cancer patients who underwent curative surgery at Changhai Hospital, Shanghai, China, from 2000 to 2004 were enrolled in this study. Histological confirmation of primary gastric endothelial carcinoma was obtained from the Department of Pathology. Other gastric tumors, such as neuroendocine tumors, lymphoma, or sarcoma, were excluded from this study. Medical records were reviewed to obtain patient data, including age at diagnosis, sex, tumor location, tumor size, anemia, blood transfusion, surgical margin status, depth of invasion, lymph node metastasis, distant metastasis, and AJCC stage.

\section{Grouping and follow-up}

Regarding to age, patients were classified into two categories according to median age (60 years old) in our group: less than 60 years old and older than 60 . Regarding tumor location, cases were divided into 4 groups according to previous report ${ }^{[13]}$ : upper, middle, lower, and whole. Regarding size, tumors were classed into 2 groups with $6 \mathrm{~cm}$ as the cutpoint: $\leqslant 6 \mathrm{~cm}$ and larger than $6 \mathrm{~cm}$. Pretreatment anemia was scored as absent or present according to previous study ${ }^{[19]}$. According to those with or without residual tumor cells at the margin of the resected tumor specimen, cases were classified into two groups: R0 and Rl. Regarding the depth of gastric wall penetration, carcinomas were classified into 4 groups: pTl (invasion of mucosa or submucosa), pT2 (invasion of muscularies propria or subserosa), pT3 (invasion of serosa), and pT4 (invasion of neighboring organs). Lymphatic permeation was scored as present or absent. Carcinoma staging was performed according to the TNM system defined by the UICC in 1997 into I , II , III , and IV .

Clinical follow-up was completed until death or until the date of last follow-up. The median followup interval for patients alive at the incised date was 37 months (range from 21 to 73 months). Postoperative mortalities, occurring within 30 days after operation, were excluded from this study.

\section{Statistical analysis}

All the statistical analyses were performed with the statistical software SPSS ${ }^{\mathrm{TM}}$ version 11.0 for Windows (SPSS, Inc., Chicago, IL). The intergroup comparisons of clinicopathological variables were made by twotailed $x^{2}$ tests for discrete variables. The KaplanMeier method was used for calculating the survival rate, and the difference between the curves was assessed with the log-rank test. A multivariate analysis of the prognostic factors was performed with the Cox proportional hazard model. The accepted level of significance was $P<0.05$.

\section{RESULTS}

\section{Incidence of transfusion}

Among 676 patients, 347 (51\%) underwent blood transfusions; the remaining $329(49 \%)$ received no transfusion. The majority of transfused patients (202 patients, $58 \%$ ) received more than $4 \mathrm{U}$ of blood, whereas 112 patients $(32 \%)$ received $3-4 \mathrm{U}$ and only 33 cases received $2 \mathrm{U}$ or less. Nearly all these patients were transfused intraoperatively, postoperatively, or both.

\section{Correlation between blood transfusion and clinicopathological parameters}

Blood transfusion was significantly associated with older age, larger tumor, upper and middle location, $\mathrm{R} 1$, and pretreatment anemia $(P<0.05)$ (Table 1). In addition, tumors in the transfused group were more advanced in depth of invasion, nodal stage, and TNM stage $(P<0.05)$ (Table 2$)$.

\section{Survival analysis}

The cumulative survival rate was significantly higher in the nontransfused than in the transfused group $(P<0.001)($ Fig. 1$)$. The median survival time was more than 50 months for the nontransfused group and 21 months for the transfused group. The median survival time was 41 months with 1-2 U, 23 months with 3-4 U, and 20 months with more than $4 \mathrm{U}$. However, no significant differences in the survival time according to the amount of transfused blood were observed $(P>0.05)$ (Fig.1).

\section{Subgroup analysis}

Strata analysis according to TNM stage, anemia, 
Table 1 Correlation between blood transfusion and clinicopathological parameters

\begin{tabular}{|c|c|c|c|}
\hline Variables & $\begin{array}{c}\text { Transfused } / n(\%) \\
(n=347)\end{array}$ & $\begin{array}{c}\text { Nontransfused } / n(\%) \\
(n=329)\end{array}$ & $P$ value \\
\hline Age/y & & & $<0.001$ \\
\hline$\leqslant 60$ & $128(37)$ & $172(52)$ & \\
\hline$>60$ & $219(63)$ & $157(48)$ & \\
\hline Sex & & & 0.410 \\
\hline Male & $243(70)$ & $234(71)$ & \\
\hline Female & $104(30)$ & $95(29)$ & \\
\hline Tumor location & & & 0.024 \\
\hline Upper third & $68(20)$ & $52(16)$ & \\
\hline Middle third & $114(33)$ & $90(27)$ & \\
\hline Lower third & $146(42)$ & $176(54)$ & \\
\hline Whole & $19(5)$ & $11(3)$ & \\
\hline Tumor size & & & $<0.001$ \\
\hline$\leqslant 6 \mathrm{~cm}$ & $197(57)$ & $259(78)$ & \\
\hline$>6 \mathrm{~cm}$ & $150(43)$ & $70(21)$ & \\
\hline $\begin{array}{l}\text { Surgical margin } \\
\text { status }\end{array}$ & & & 0.013 \\
\hline No & $307(89)$ & $309(94)$ & \\
\hline Yes & $39(11)$ & $19(6)$ & \\
\hline Anemia & & & $<0.001$ \\
\hline Yes & $208(60)$ & $85(26)$ & \\
\hline No & $139(40)$ & $244(74)$ & \\
\hline
\end{tabular}

Table 2 Tumor, node metastasis, and stage between transfused and nontransfused patients

\begin{tabular}{lccc}
\hline Variables & $\begin{array}{c}\text { Transfused } / n(\%) \\
(n=347)\end{array}$ & $\begin{array}{c}\text { Nontransfused } / n(\%) \\
n=329\end{array}$ & $P$ value \\
\hline Depth of invasion & & & $<0.001$ \\
T1 & $24(7)$ & $69(21)$ & \\
T2 & $42(12)$ & $61(18)$ & \\
T3 & $255(74)$ & $174(53)$ & \\
T4 & $26(7)$ & $25(8)$ & \\
Nodal involvement & & & $<0.001$ \\
N0 & $102(29)$ & $127(39)$ & \\
N1 & $123(35)$ & $120(37)$ & \\
N2 & $81(23)$ & $70(21)$ & \\
N3 & $41(12)$ & $12(4)$ & \\
TNM & & & $<0.001$ \\
I & $44(13)$ & $95(29)$ & \\
II & $69(20)$ & $67(20)$ & \\
III & $165(48)$ & $140(43)$ & \\
IV & $69(20)$ & $27(8)$ & \\
\hline
\end{tabular}

and tumor size was carried out. Subgroup analysis of prognosis according to stage, showed significant differences in stage II and III , but not in stage I and IV , between the transfused and nontransfused groups ( $>67$ months for nontransfused groups vs. $>62$ months for transfused groups in stage I,$P=0.2070$; $>58$ months vs. 58 months in stage II , $P=0.0064$; 29 months vs. 20 months in stage III , $P=0.0071$; 16 months vs. 10 months in stage $\mathrm{IV}, P=0.3235$ ) (Fig.2). A significant difference between the transfused and nontransfused groups could be observed in the strata of tumor size ( $>52$ months $v s .30$ months in patients with tumor $\leqslant 6 \mathrm{~cm}, P<0.001 ; 43$ months $v s$. 17 months in patients with tumor $>6 \mathrm{~cm}, P=0.0019)$ (Fig.3). Patients with or without anemia in the nontransfused groups both had a better survival time than those in the transfused groups ( $>52$ months for nontransfused groups $v s .29$ months for transfused groups in patients without anemia, $P<0.001 ; 39$ months vs. 20 months in patients with anemia, $P=0.0072$ ) (Fig.4).

\section{Regression analysis}

The multivariate analysis was performed by Cox's proportional hazard model. The transfusion was an independent prognostic factor $(P=0.002)$, as well as older age, depth of invasion, LN metastases, and TNM stage (Table3).

\section{DISCUSSION}

The impact of perioperative blood transfusion on the survival of cancer patients remains a contradictory issue. There have been several reports on the adverse effect of transfusion in patients undergoing surgery for cancer. The most possible explanation is the role of nonspecific immunosuppression, probably due to impaired mitogen-induced blastogenesis, decreased NK cells activity ${ }^{[20]}$, increased activities of suppressor $\mathrm{T}$ lymphocytes, and stimulated anti-idiotypic antibody production $^{[21]}$. However, other studies provided an opposite opinion, that there was no causal relationship between blood transfusions and poor survival rates. They point out that it is not the transfusion itself that results in poor prognosis in transfused patients, but rather it is a combination of several other factors, such

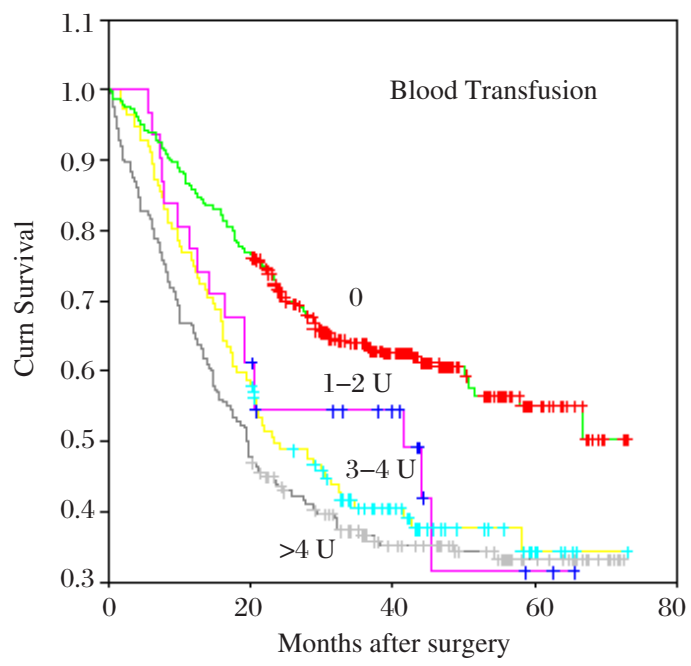

Fig. 1. Cumulative survival curves according to the amount of transfusion. Significant difference could be observed between the nontransfused group and transfused group. But no significant relationship was found between the amount of transfused blood and prognosis. 

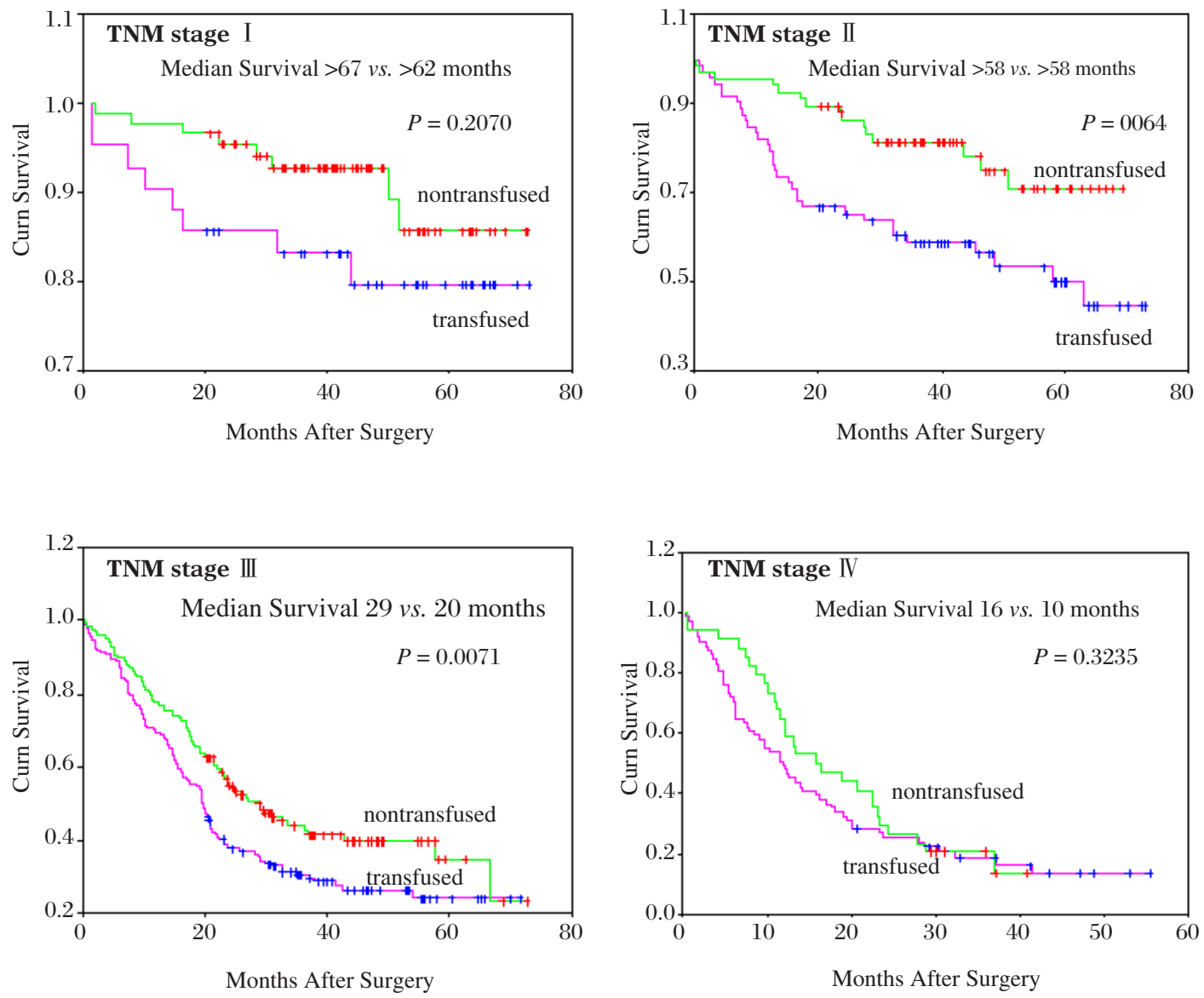

Fig. 2. Subgroup analysis of prognosis according to stage showed significant differences in stage II and III, not in stage I and IV between the transfused and nontransfused groups
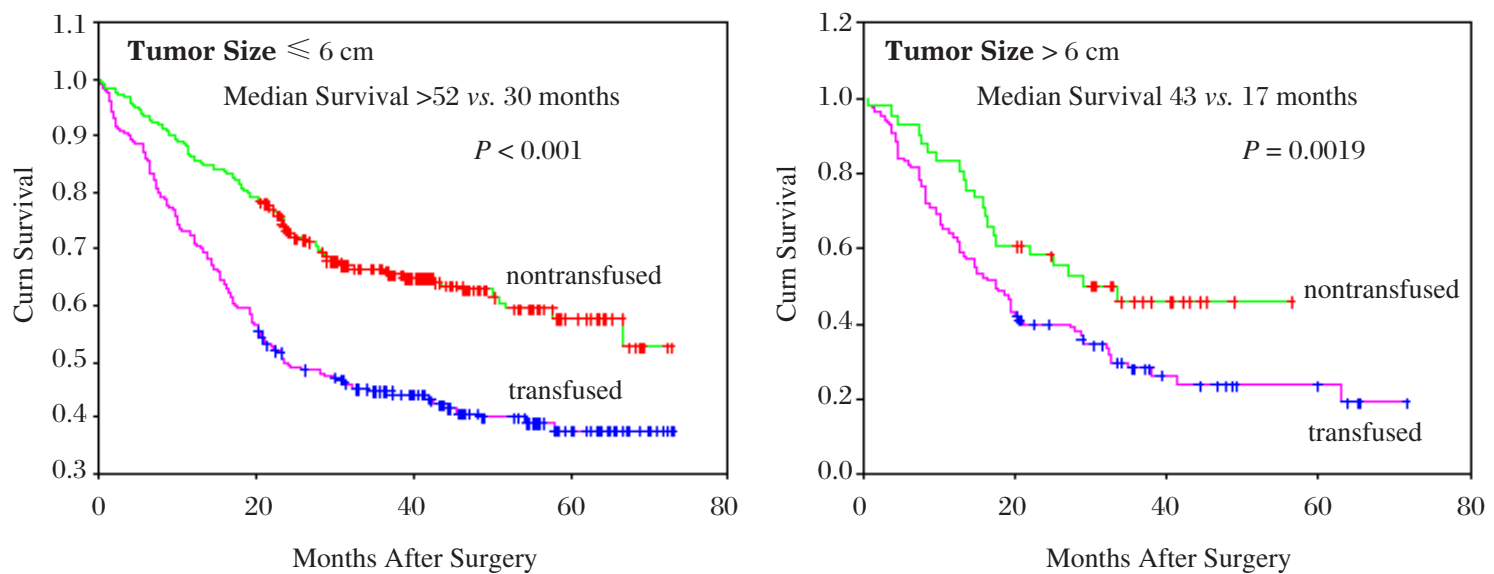

Fig. 3. Subgroup analysis of prognosis according to tumor size showed significant differences between the transfused and nontransfused groups whether tumor size $\leqslant 6 \mathrm{~cm}$ or $>6 \mathrm{~cm}$ 

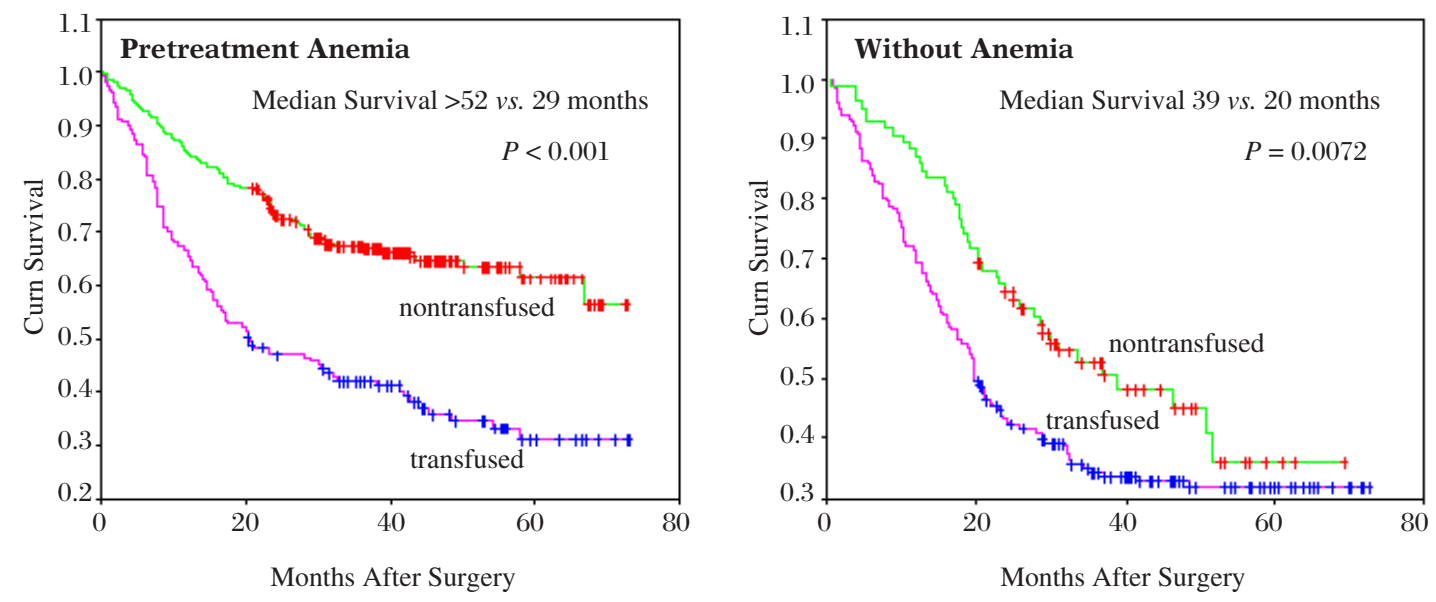

Fig. 4. Subgroup analysis of prognosis according to anemia or without anemia both showed significant differences between the transfused and nontransfused groups.

Table 3 Cox's proportional hazard model analysis of prognostic factors

\begin{tabular}{|c|c|c|c|c|c|c|}
\hline Covariate (observed value) & Beta & SE & Wald & $\operatorname{Exp}(B)$ & $95 \% \mathrm{CI}$ & $P$ value \\
\hline Age $(y)(\leqslant 60 v s .>60)$ & -0.467 & 0.116 & 16.316 & 0.627 & $0.500-0.786$ & $<0.001$ \\
\hline Sex (male $v s$. female) & -0.098 & 0.131 & 0.640 & 0.907 & $0.980-1.640$ & 0.424 \\
\hline Transfusion (no vs. yes) & -0.378 & 0.122 & 9.513 & 0.685 & $0.539-0.871$ & 0.002 \\
\hline Location (lower and mid body $v s$. upper body and whole) & 0.237 & 0.131 & 3.269 & 1.268 & $0.980-1.640$ & 0.071 \\
\hline Anemia (no vs. yes) & -0.025 & 0.122 & 0.041 & 0.839 & $0.768-1.239$ & 0.839 \\
\hline Margin status (no vs. yes) & -0.234 & 0.184 & 1.612 & 0.792 & $0.552-1.136$ & 0.204 \\
\hline Size $(\leqslant 6 \mathrm{~cm} v s .>6 \mathrm{~cm})$ & -0.177 & 0.122 & 2.099 & 0.838 & $0.660-1.064$ & 0.147 \\
\hline Gastric wall invasion (T1/T2 vs. T3/T4) & -0.746 & 0.187 & 15.866 & 0.474 & $0.329-0.685$ & $<0.001$ \\
\hline Nodal involvement (negative $v s$. positive) & -1.335 & 0.163 & 67.199 & 0.263 & $0.191-0.362$ & $<0.001$ \\
\hline TNM stage ( I / II vs. III / IV ) & 0.292 & 0.138 & 4.482 & 1.339 & $1.022-1.755$ & 0.034 \\
\hline
\end{tabular}

as cancer bleeding, anemia caused by long-standing malnutrition, larger tumor size, and advanced disease stage ${ }^{[22,23]}$. Previous studies proposed that factors like inadequate study design, inadequate eligibility criteria, or small sample size may have be contributing to the confusing results. However, many retrospective and prospective randomized studies from world leading labs also got contradictory results. In their reports, sample size did not seem to be a problem since most studies recruited hundreds, even thousands of cases. Thus, the contradiction may have arisen from inadequate statistical analysis, or the different ethnic and geographic origin of patients. In this study, we investigated the role of blood transfusions on the survival of gastric cancer patients from China.

The main findings of this study are that (1) transfusion is an independent risk factor for poor prognosis and that (2) adverse effects of perioperative transfusion are significantly associated with patients with stage II and III gastric cancer and that (3) tumor size and pretreatment anemia don't affect the prognostic value of transfusion on gastric cancer patients.

Clinicopathological factors including older age, larger tumor, surgical margin, and pretreatment anemia were associated with transfusion in our study. The above factors were more frequently observed in the advanced stage. Moreover, blood transfusion was also frequently seen in advanced $\mathrm{pT}, \mathrm{pN}$, and TNM stage, similar to previous studies ${ }^{[13]}$. These results proved that perioperative blood transfusion mainly took place in advanced tumor stage, suggesting its necessity in benefiting some patients. The following study should be focused on subgroup analysis according to the pathologic stage, tumor size, or even pretreatment anemia.

This study showed a significantly poor survival rate in the transfused group compared with the nontransfused group. The comparison in stages II and III revealed significant differences in the median survival time, whereas those in stages I and IV did not. Hyung et $a l .{ }^{[13]}$ observed adverse effects of perioperative transfusion on patients with stage III and IV gastric cancer, but not with stage I and II gastric cancer. Heiss et al. ${ }^{[10]}$ reported that the prognostic effect of transfusion is mediated through an impact on minimal residual disease in resected cancer patients. They believed that trans- 
fusion-associated immunosuppression may cause the progression of metastatic foci and failure to remove circulating cancer cells, and cells in bone marrow in advanced stage cases. However, the further the stage advances, the more compounding factors come in to play, such as longer time of operation, total gastrectomy, more extended lymphadenectomy, and the poor nutritional status of patients. These findings may partly support our results that transfused-related immune modulation, significantly affects the poor outcome of gastric patients in stages II and III , but not in stages IV and I . In stage IV , the circumstances mentioned above necessitating transfusions may be more important prognostic factors ${ }^{[16]}$. Stage I , in contrast to stage IV , may have favorable factors, such as local gastrectomy, reduced operation time, and good nutritional status, making these patients recover more easily from the operation and the disease. From the above results, we concluded that perioperative blood transfusion itself had a statistically significant influence on the prognosis of patients with gastric cancer.

Multiple studies revealed that tumor size was an important prognostic factor after curative gastrectomy for advanced gastric cancer. Sugezawa A et al. ${ }^{[23]}$ reported that the survival of patients in their transfused group was significantly lower than that in nontransfused group, and the transfused group of patients was found to have tumors that were larger than those in the non-transfused group. They concluded that this adverse effect in the transfused patients was probably attributable to the larger size of their tumors. In our study, subgroup analyses of prognosis according to tumor size showed significant differences between the transfused and nontransfused groups both in patients with tumor sizes less than $6 \mathrm{~cm}$ and in those with tumor sizes larger than $6 \mathrm{~cm}$. This finding revealed again that transfusion was shown to be an independent risk factor for poor prognosis.

A negative correlation between anemia and outcome has been demonstrated in various cancers. Shen $\mathrm{JG}$ et al. ${ }^{[19]}$ reported that pretreatment anemia had an independent relationship to the long-term survival of patients with stage I and II gastric cancer. Perioperative blood transfusion frequently happened in patients with pretreatment anemia in this study. Subgroup analyses of prognosis according to patients being with or without anemia at the time of operation were also carried out. Whether with or without anemia, patients in nontransfused groups had a longer median survival time than those in transfused groups. The difference between transfusion and anemia further supported the significance of transfusion on the survival of gastric cancer.

In conclusion, it is the advanced stage of tumor and extended surgery that necessitate perioperative blood transfusion. However, transfusion itself is an unfavorable prognostic factor for patients with gastric cancer, especially for those with stage II and III gastric cancer. In addition, the parameters like tumor staging, tumor size, and pretreatment anemia do not seem to affect its prognostic value.

\section{References}

[1] Cata JP, Gottumukkala V. Blood transfusion practices in cancer surgery. Indian J Anaesth, 2014, 58(5):637-42.

[2] Lopez-Andrade Jurado A, Almazan Duro A, Martin Ruiz JL, et al. Immune response in the surgical patient: effect of anesthesia and blood transfusion. Rev Esp Anestesiol Reanim, 2000, 47(2):67-80.

[3] Yamamoto J, Kosuge T, Takayama T, et al. Perioperative blood transfusion promotes recurrence of hepatocellular carcinoma after hepatectomy. Surgery, 1994, 115(3):303-9.

[4] Tartter PI, Burrows L, Papatestas AE, et al. Perioperative blood transfusion has prognostic significance for breast cancer. Surgery, 1985, 97(2):225-30.

[5] Taniguchi Y, Okura M. Prognostic significance of perioperative blood transfusion in oral cavity squamous cell carcinoma. Head Neck, 2003, 25(11):931-6.

[6] Luan H, Ye F, Wu L, et al. Perioperative blood transfusion adversely affects prognosis after resection of lung cancer: a systematic review and a meta-analysis. $B M C$ Surg, 2014, 14:34.

[7] Chesi R, Cazzola A, Bacci G, et al. Effect of perioperative transfusions on survival in osteosarcoma treated by multimodal therapy. Cancer, 1989, 64(8):1727-37.

[8] Kosumi K, Baba Y, Harada K, et al. Perioperative blood transfusion, age at surgery, and prognosis in a database of 526 upper gastrointestinal cancers. Dig Surg, 2015, 32(6):445-53.

[9] Yeoh TY, Scavonetto F, Weingarten TN, et al. Perioperative allogeneic nonleukoreduced blood transfusion and prostate cancer outcomes after radical prostatectomy. Transfusion, 2014, 54(9):2175-81.

[10] Heiss MM, Allgayer H, Gruetzner KU, et al. Prognostic influence of blood transfusion on minimal residual disease in resected gastric cancer patients. Anticancer Res, 1997, 17(4A):2657-61.

[11] Dresner SM, Lamb PJ, Shenfine J, et al. Prognostic significance of peri-operative blood transfusion following radical resection for oesophageal carcinoma. Eur J Surg Oncol, 2000, 26(5):492-7.

[12] Murata N, Idezuki Y, Konishi T, et al. Influence of perioperative blood transfusion on the prognosis of patients with gastric cancer receiving anticancer chemotherapy. Gastric Cancer, 2000, 3(1):24-7.

[13] Hyung WJ, Noh SH, Shin DW, et al. Adverse effects of perioperative transfusion on patients with stage III and 
IV gastric cancer. Ann Surg Oncol, 2002, 9(1):5-12.

[14] Sun C, Wang Y, Yao HS, et al. Allogeneic blood transfusion and the prognosis of gastric cancer patients: systematic review and meta-analysis. Int J Surg, 2015, 13:102-10.

[15] Squires MH 3rd, Kooby DA, Poultsides GA, et al. Effect of perioperative transfusion on recurrence and survival after gastric cancer resection: A 7-institution analysis of 765 patients from the US Gastric Cancer Collaborative. J Am Coll Surg, 2015, 221(3):767-77.

[16] Choi JH, Chung HC, Yoo NC, et al. Perioperative blood transfusions and prognosis in patients with curatively resected locally advanced gastric cancer. Oncology, 1995, 52(2):170-5.

[17] Sanchez-Bueno F, Garcia-Marcilla JA, Perez-Abad $\mathrm{JM}$, et al. Does perioperative blood transfusion influence long-term prognosis of gastric cancer? Dig Dis Sci, 1997, 42(10):2072-6.

[18] Cui J, Deng J, Ding X, et al. Blood transfusion does not affect survival of gastric cancer patients. J Surg Res,
2016, 200(1):98-104.

[19] Shen JG, Cheong JH, Hyung WJ,et al. Pretreatment anemia is associated with poorer survival in patients with stage I and II gastric cancer. J Surg Oncol, 2005, 91(2):126-30.

[20] Maeta M, Shimizu N, Oka A, et al. Perioperative allogeneic blood transfusion exacerbates surgical stressinduced postoperative immunosuppression and has a negative effect on prognosis in patients with gastric cancer. J Surg Oncol, 1994, 55(3):149-53.

[21] Wu HS, Little AG. Perioperative blood transfusions and cancer recurrence. J Clin Oncol, 1988, 6(8):1348-54.

[22] Kampschoer GH, Maruyama K, Sasako M, et al. The effects of blood transfusion on the prognosis of patients with gastric cancer. World J Surg, 1989, 13(5):637-43.

[23] Sugezawa A, Kaibara N, Sumi K, et al. Blood transfusion and the prognosis of patients with gastric cancer. $J$ Surg Oncol, 1989, 42(2):113-6.

(Received 20 April 2017, Revised 25 May 2017, Accepted 11 June 2017) 